\title{
Cataract surgery and quality of life in patients with age related macular degeneration
}

\author{
M Lundström, K G Brege, I Florén, B Lundh, U Stenevi, W Thorburn
}

Br J Ophthalmol 2002;86:1330-1335

Series editors: $G$ and $M$ Brown

See end of article for authors' affiliations

Correspondence to: Dr Mats Lundström; Department of Ophthalmology, Blekinge Hospital, SE-371 85 Karlskrona, Sweden mats.lundstrom@ltblekinge.se

Accepted for publication 16 July 2002
Background: The coexistence of cataract and age related macular degeneration (AMD) is not unusual, especially in the very elderly. The outcome of cataract surgery in these cases depends on the effect of $A M D$ on vision. In this study the authors have compared the outcome of cataract patients with AMD to that of cataract patients with no vision threatening ocular comorbidity, and analysed possible predictors of good or poor outcome.

Methods: An observational prospective study on consecutive cases operated for cataract during 1 month at six surgical departments affiliated to the Swedish National Cataract Register (NCR). Data were collected according to the protocol of NCR and subjects completed the Catquest questionnaire before and 6 months after surgery. 90 subjects with AMD were compared to 335 subjects with no sight threatening ocular comorbidity.

Results: Difficulties in performing various daily life activities improved significantly for AMD subjects after surgery ( $p<0.001$, Wilcoxon signed rank test). Satisfaction with vision also improved significantly after surgery ( $p<0.001$, Wilcoxon signed rank test). Activity level and independence were unchanged. Subjects with no ocular comorbidity had a still better outcome. The most important variable related to a good self assessed functional outcome was postoperative visual acuity irrespective of the presence of $A M D$. AMD subjects scheduled for second eye surgery and $A M D$ subjects dissatisfied with their vision before surgery had a poorer outcome.

Conclusion: Subjects with various stages of dry AMD and cataract improved their self assessed visual function and satisfaction with vision significantly after cataract extraction.
$\mathrm{T}$ he number of cataract extractions is increasing in many countries. ${ }^{1-3}$ There are several reasons for an increasing surgical volume such as a higher frequency of second eye surgery, ${ }^{4}$ faster surgery with fewer risks, ${ }^{5}$ and an ageing society. ${ }^{5}$ A substantial number of patients undergoing a cataract extraction also have a coexisting eye disease that may influence the outcome of surgery. The number of patients with an ocular comorbidity in the eye to be operated on will probably increase as the indications for cataract surgery become wider and the surgery rate rises. In a recent cataract surgery survey including a number of European ophthalmic departments an ocular comorbidity in the eye to be operated on was found in $37.5 \%$ of all cases. ${ }^{\circ}$

An important outcome measure in cataract surgery is the patients' self assessed visual function. ${ }^{7}$ Several studies have disclosed that the existence of an ocular comorbidity in the eye to be operated on for cataract is related to a poor self assessed functional outcome. ${ }^{8-10}$ A large difference between expected and actual postoperative functioning in patients with an ocular comorbidity has also been reported. ${ }^{11}$ Age related macular degeneration (AMD) is a common ocular comorbidity in patients with cataract. ${ }^{68}$

In a European survey of patients undergoing a cataract extraction the mean frequency of AMD postoperatively was $13.7 \%{ }^{6}$ With rapidly ageing populations and greater life expectancy, the number of patients with both cataract and AMD will inevitably increase. ${ }^{12}$

A few studies have shown that patients with AMD and cataract benefit from a cataract extraction. ${ }^{13}{ }^{14}$ What subset, if any, of patients with AMD is likely to benefit from a cataract extraction is poorly understood. Important questions are, therefore, is cataract surgery justified in patients with AMD and are there subgroups of patients with AMD that will benefit more than others?

The Swedish National Cataract Register $(\mathrm{NCR})^{115}$ has been registering the occurrence of a macular degeneration in the eye to be operated on since 1999. In this study, we have analysed the outcomes of cataract surgery for patients with AMD at six surgical departments.

We aimed to investigate the self assessed functional outcome of cataract surgery for patients with AMD in the operated eye (AMD subjects) and to make a comparison with patients without any coexisting vision threatening eye disease in the operated eye (control subjects).

\section{METHODS}

The following data were collected prospectively from each patient.

- Preoperative data: Clinic, age, sex, visual acuity in both eyes, first or second eye surgery, date on waiting list, known sight threatening ocular comorbidity in the eye to be operated on, keratometry values of the eye to be operated on, and target refraction.

- Peroperative data: Date of surgery, type of surgery, and type of IOL.

- Postoperative data: Date of follow up, keratometry values of the operated eye, visual acuity in both eyes, refraction of the operated eye, and occurrence of a sight threatening ocular comorbidity in the operated eye.

Visual acuity (VA) was obtained by letter charts and expressed as decimal notations. VA of 0.1 or less was detailed in the following steps according to the medical records: 0.01, 0.02, 0.04, $0.06,0.08$, and 0.1 . The decimal notations were converted mathematically to $\log$ MAR units ${ }^{16}$ for some of the analyses (mean values and description of the magnitude of improvement of VA). Hand movement vision and perception of light were assumed to be equivalent to $\log$ MAR 2.0.

All patients completed the Catquest ${ }^{17}{ }^{18}$ questionnaire before and 6 months after surgery. The Catquest contains questions about perceived difficulties in daily life, activity level in daily 
Table 1 Number of subjects operated during the study month at six surgical departments

\begin{tabular}{|c|c|c|c|c|c|c|c|}
\hline & \multicolumn{7}{|c|}{ Clinic } \\
\hline & 3 & 6 & 13 & 16 & 20 & 22 & All \\
\hline Number of operated subjects with follow up & 146 & 133 & 106 & 122 & 160 & 104 & 771 \\
\hline With AMD preoperatively & 39 & 38 & 38 & 24 & 28 & 24 & $191(24.8 \%)$ \\
\hline Number of subjects with follow up and completed Catquest & 102 & 102 & 95 & 87 & 113 & 79 & 578 \\
\hline With verified $A M D$ & 23 & 24 & 21 & 7 & 9 & 6 & $90(15.6 \%)$ \\
\hline With other sight threatening ocular comorbidity except AMD & 27 & 32 & 18 & 26 & 32 & 18 & $153(26.4 \%)$ \\
\hline Without any sight threatening ocular comorbidity (control) & 52 & 46 & 56 & 54 & 72 & 55 & $335(58 \%)$ \\
\hline
\end{tabular}

life, cataract symptoms, satisfaction with vision, and independent living. No question refers to the operation itself or to satisfaction with care. The responses are evaluated by means of a decision model. Briefly, an improvement in perceived disabilities, cataract symptoms, and activity level is called a "very good benefit"; improvement in perceived disabilities and cataract symptoms is called a "good benefit"; improvement in perceived disabilities only is called a "moderate benefit"; status quo in perceived disabilities is called "questionable benefit"; and a deterioration in perceived disabilities is called "no benefit."

The NCR data were checked in the medical records of each patient concerning the diagnosis and type of AMD and preoperative and postoperative visual acuity (VA). The NCR data were on average collected 2 months before the completion of the postoperative questionnaire. The medical record check adjusted in most cases this discrepancy of dates. The diagnosis of AMD was entirely clinical, made by the surgeon and based on morphological appearance of macula and visual functioning.

Relation between ranked data was tested using MannWhitney $U$ test. Relation between various independent variables and dependent variables was tested using binary logistic regression analysis. The relation between categorical data was tested using the $\chi^{2}$ test and test of relation between a pair of related variables was performed using Wilcoxon signed rank test.

\section{PATIENTS}

Consecutive patients operated on during March 1999 at six surgical units were included in the study. Patients with the diagnosis of an AMD before surgery and verified after surgery were included as study patients. Patients with no known vision threatening ocular comorbidity before and after surgery were included as controls. Patients with coexisting eye diseases other than AMD in the operated eye were excluded. Altogether, 771 subjects were operated on at the six clinics during the study month. The number of subjects with surgical outcome data and completed Catquest before and after surgery was 578 (75\% of the total number of patients). The most frequent reason for a dropout was an incomplete

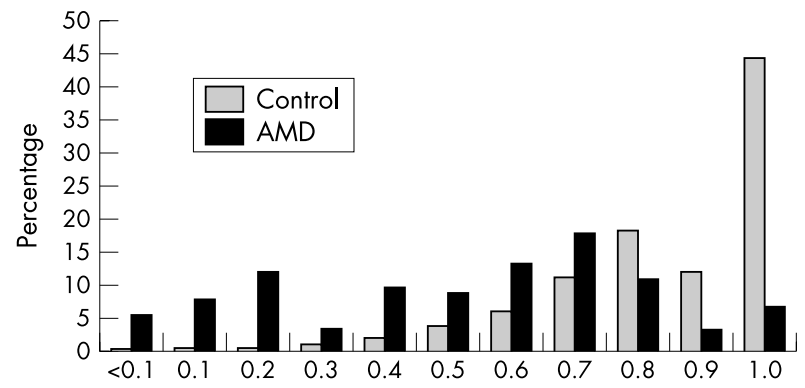

Figure 1 Postoperative visual acuity (decimal notation) for AMD subjects and control subjects. questionnaire postoperatively (Table 1). Another reason for exclusion was cataract surgery in the fellow eye during follow up. Ninety patients had a verified AMD and 335 patients had no vision threatening ocular comorbidity in the operated eye (Table 1). Excluded from further analysis were 153 patients with another vision threatening ocular comorbidity in the operated eye. All patients with AMD had a dry AMD. They represented various stages of dry AMD from drusen, pigments, and small atrophies to large geographic scars. The most frequent surgical procedure was phacoemulsification $(97.2 \%)$ and all patients (AMD subjects and control subjects) received an IOL.

Patients with AMD were significantly older than patients with no ocular comorbidity (mean age 82.4 years $v 74.8$ years, $\mathrm{p}<0.001 t$ test). The sex distribution was about the same for both groups (73.3\% female subjects in the AMD group $v 69.3 \%$ in the control group, $\left.\mathrm{p}=0.453, \chi^{2}\right)$. Second eye surgery was significantly more frequent in the AMD group $(51.1 \%$ second eye surgeries $v 33.7 \%, \mathrm{p}=0.002, \chi^{2}$ ).

\section{RESULTS}

The postoperative visual acuity is shown in Figure 1. Subjects with no ocular comorbidity had significantly better visual outcome than subjects with AMD $(\mathrm{p}<.001$, Mann-Whitney U tests). The median VA (decimal notation) in the surgery eye postoperatively was 0.9 for controls and 0.6 for AMD subjects and the mean VA (logMAR) was 0.10 and 0.42 , respectively.

The functional outcome as defined by Catquest for AMD subjects and controls is shown in Table 2 and satisfaction with vision is shown in Table 3. The functional outcome signifies the difference between preoperative and postoperative perceived difficulties in daily life and satisfaction with vision signifies the self assessed satisfaction at the time of questioning. The functional outcome was significantly better in the control group $(\mathrm{p}<0.001$, Mann-Whitney U test) and so was satisfaction with vision ( $p=0.001$, Mann-Whitney $U$ test $)$. In the control group $89 \%$ of the subjects perceived fewer disabilities in performing daily life activities after surgery compared to $75.6 \%$ in the AMD group; $86.2 \%$ of the control group and $66.6 \%$ of the AMD group were satisfied with their vision. Preoperatively, there was no significant difference in satisfaction with vision

Table 2 Outcome of cataract surgery as defined by Catquest for AMD subjects and control subjects

\begin{tabular}{llll}
\hline \multirow{2}{*}{ Benefit level } & \multicolumn{3}{l}{ Group of subjects* } \\
\cline { 2 - 4 } & Meaning & Control & AMD \\
\hline Very good benefit & Improved & $46.6 \%$ & $25.6 \%$ \\
Good benefit & Improved & $7.2 \%$ & $12.2 \%$ \\
Moderate benefit & Improved & $35.2 \%$ & $37.8 \%$ \\
Questionable benefit & Status quo & $5.7 \%$ & $8.9 \%$ \\
No benefit & Worse & $5.4 \%$ & $15.6 \%$ \\
\hline
\end{tabular}

*Test of relation between groups of subjects: $p<0.001$ (Mann-Whitney U) 
Table 3 Satisfaction with vision after cataract surgery for AMD subjects and control subjects

\begin{tabular}{llll}
\hline \multirow{4}{*}{ Satisfaction } & \multicolumn{3}{l}{ Group of subjects* } \\
\cline { 2 - 4 } & Meaning & Control & AMD \\
\hline Very satisfied & Satisfied & $54.3 \%$ & $34.4 \%$ \\
Rather satisfied & Satisfied & $31.9 \%$ & $32.2 \%$ \\
Rather dissatisfied & Dissatisfied & $7.5 \%$ & $13.3 \%$ \\
Very dissatisfied & Dissatisfied & $2.4 \%$ & $12.2 \%$ \\
Don't know/no answer & & $3.9 \%$ & $7.7 \%$ \\
\hline \multirow{2}{*}{ *Test of relation between groups of subjects: $\mathrm{p}=0.001$} & \\
(Mann-Whitney U). & & \\
\hline
\end{tabular}

between subjects with AMD and those without comorbidity (28.9\% satisfied in the AMD group and $23.7 \%$ satisfied in the control group; $p=0.940$, Mann-Whitney U test).

The AMD group was significantly older than the control group. This difference in age may partly explain the difference in outcomes. Therefore, the functional outcome was tested on a subgroup aged 80 or older. In this subgroup there were 124 subjects with no ocular comorbidity and 64 subjects with AMD. The mean age for the AMD subgroup was 85.1 years and for the control subgroup 84.2 years $(p=0.093, t$ test). The functional outcome in benefit levels as defined by Catquest was significantly better in the control group than in the AMD group ( $87.9 \%$ improved in the control group $v 71.9 \%$ improved in the AMD group; $p=0.013$, Mann-Whitney $U$ ), and satisfaction with vision was also significantly better in the control group ( $87.1 \%$ satisfied in the control group $v 59.4 \%$ satisfied in the AMD group; $\mathrm{p}=0.002$, Mann-Whitney U).

The outcome for all subjects was dichotomised in three different ways:

Perceived difficulties in performing daily life activities:

(1) $>$ improved or status quo $=v>$ worse $=$

(2) $>$ improved $=v>$ worse or status quo $=$

Satisfaction with vision:

(3) $>$ satisfied $=v>$ not satisfied $=$.

Each dichotomised outcome was tested as a dependent variable in a logistic regression analysis. Independent variables were age, sex, second eye surgery, AMD, preoperative disability score, preoperative activity score, preoperative global score, VA surgery eye preoperative, VA fellow eye preoperative, postoperative VA surgery eye. The variable most strongly related to the outcome was postoperative visual acuity in the operated eye $(p<0.001$ for all three dependent variables $)$ and

Table 4 Relation between preoperatively known variables and the outcomes. Only variables found to be significantly related to some of the outcomes are included in the table.

\begin{tabular}{lll}
\hline \multirow{2}{*}{ Dependent variable } & $\begin{array}{l}\text { Independent } \\
\text { variables }\end{array}$ & $\mathrm{p}$ Value* \\
\hline 1 Improved or status quo $v$ worse & VA, fellow eye & 0.022 \\
& AMD & 0.038 \\
& Disability score & 0.449 \\
& Sex & 0.541 \\
2 Improved $v$ status quo or worse & VA, fellow eye & 0.010 \\
& AMD & 0.012 \\
& Disability score & 0.004 \\
\multirow{2}{*}{ Satisfied $v$ not satisfied } & Sex & 0.921 \\
& VA, fellow eye & 0.049 \\
& AMD & 0.013 \\
& Disability score & 0.027 \\
*Logistic regression. & Sex & 0.031 \\
\hline \multirow{2}{*}{. } & \\
\hline
\end{tabular}

Table 5 Relation between outcome of cataract surgery and improved visual acuity for AMD subjects and control subjects. Improvement of visual acuity was defined as one line or more on the letter chart

\begin{tabular}{llll}
\hline Patient group & Control & AMD & p Value* \\
\hline All patients ( $\mathrm{n}=425$ ) & 335 & 90 & \\
$\quad$ Improved or status quo (\%) & 94.6 & 84.4 & 0.001 \\
$\quad$ Improved (\%) & 89.0 & 75.6 & 0.001 \\
$\quad$ Satisfied (\%) & 86.9 & 70.0 & $<0.001$ \\
& & & \\
Patients with improved visual & 322 & 76 & \\
acuity after surgery ( $=398$ ) & & & \\
$\quad$ Improved or status quo (\%) & 95.0 & 85.5 & 0.003 \\
$\quad$ Improved (\%) & 89.1 & 78.9 & 0.017 \\
$\quad$ Satisfied (\%) & 87.3 & 73.7 & 0.003 \\
$\quad$ & & 14 & \\
Patients with worse visual acuity & 13 & & \\
or status quo after surgery (n = & & & \\
27 Improved or status quo (\%) & 84.6 & 78.6 & 0.686 \\
$\quad$ Improved (\%) & 84.6 & 57.1 & 0.118 \\
$\quad$ Satisfied (\%) & 76.9 & 50.0 & 0.148 \\
\hline * ${ }^{2}$ test. & & & \\
\hline
\end{tabular}

the occurrence of AMD was not related to any of the outcomes. If postoperative VA in the operated eye was excluded as an independent variable in the analyses and only preoperatively known variables were tested the occurrence of AMD was related to the outcomes (Table 4).

In Table 5 the dichotomised outcomes are demonstrated for all patients, both those who have improved their vision after surgery and for those who have not improved after surgery. Improvement of visual acuity was defined as one line or more on the letter chart. The outcome was significantly better for subjects with no ocular comorbidity compared to subjects with AMD when visual acuity improved after surgery. When there was no improvement in VA after surgery the difference between the groups in functional outcome and satisfaction was even greater, but owing to the low number of cases statistical significance was not reached (Table 5). When all subjects were grouped in three categories depending on postoperative visual acuity in the operated eye $(0.01-0.3,0.4-0.7,0.8-1.0)$, there was no statistical significant difference in functional outcome and satisfaction with vision between the control group and the AMD group (Table 6).

The magnitude of improvement of VA ( $\log M A R)$ in the operated eye was tested for a relation to the dichotomised outcome for control subjects and AMD subjects using the Mann-Whitney U test. No significant relation was found for any of groups and outcomes.

Though the AMD group had a poorer outcome than the control group, the AMD subjects showed a significant improvement in all recorded visual function variables after

Table 6 Test of relation between the outcomes of AMD subjects and control subjects. All patients grouped by their postoperative VA in the operated eye

\begin{tabular}{|c|c|c|c|}
\hline \multirow{4}{*}{$\begin{array}{l}\text { Outcome } \\
\text { VA, decimal notation } \\
\text { VA, logMAR units } \\
\mathrm{N} \text { o }\end{array}$} & \multicolumn{3}{|c|}{ Postoperative VA, operated eye } \\
\hline & $0.01-0.3$ & $0.4-0.7$ & $0.8-1.0$ \\
\hline & $2-0.5$ & $0.4-0.2$ & $0.1-0$ \\
\hline & 35 & 126 & 264 \\
\hline & \multicolumn{3}{|l|}{$\mathrm{p}$ value * } \\
\hline Improved or status quo $v$ worse & 0.735 & 0.790 & 0.178 \\
\hline Improved $v$ statusquo or worse & 0.331 & 0.696 & 0.738 \\
\hline Satisfied $v$ not satisfied & 0.589 & 0.497 & 0.570 \\
\hline
\end{tabular}


Table 7 Perceived difficulties in performing 7 daily life activities before and after cataract surgery. AMD subjects compared to control subjects. In the table the scores for each activity and group of subjects are shown as mean values, but in the test of relation a non-parametric test for ranked data is used (Mann-Whitney $U$ test)

\begin{tabular}{|c|c|c|c|c|c|c|}
\hline \multirow[b]{2}{*}{ Daily life activity } & \multicolumn{3}{|c|}{ Mean score before surgery } & \multicolumn{3}{|c|}{ Mean score after surgery } \\
\hline & Control & AMD & p Value* & Control & AMD & $\mathrm{p}$ Value* \\
\hline Reading paper text & 2.31 & 2.67 & 0.041 & 1.37 & 1.91 & $<0.001$ \\
\hline Recognising faces & 2.16 & 2.21 & 0.770 & 1.27 & 1.67 & $<0.001$ \\
\hline Seeing prices & 2.38 & 2.78 & 0.015 & 1.43 & 2.00 & $<0.001$ \\
\hline Seeing to walk on uneven ground & 2.25 & 2.36 & 0.541 & 1.53 & 2.08 & $<0.001$ \\
\hline Seeing needlework & 2.54 & 2.91 & 0.123 & 1.36 & 2.28 & $<0.001$ \\
\hline Seeing text on TV & 2.18 & 2.51 & 0.045 & 1.28 & 1.87 & $<0.001$ \\
\hline Seeing to perform a preferred hobby & 2.38 & 2.34 & 0.756 & 1.36 & 1.95 & $<0.001$ \\
\hline
\end{tabular}

surgery $(\mathrm{p}<0.001$, Wilcoxon signed ranks test). Visual acuity was improved in 76 subjects $(84.4 \%)$, the disability score as defined by Catquest was improved in 62 subjects $(68.9 \%)$, and satisfaction with vision was improved in 58 subjects $(64.4 \%)$.

The total activity score ( six daily life activities) as defined by Catquest ${ }^{17}$ was significantly better for the control group compared to the AMD group both before $(p=0.008$, MannWhitney $U)$ and after ( $p=0.003$, Mann-Whitney U) surgery. If the comparison is confined to subjects 80 years or older the difference in activity level disappears $(\mathrm{p}=0.256$ and $\mathrm{p}=0.120$, respectively).

The total disability score (seven daily life activities) as defined by Catquest ${ }^{17}$ did not differ significantly before surgery between the AMD group and the control group $(p=0.096$, Mann-Whitney U). For subjects 80 years or older, however, the AMD subjects perceived significantly more difficulties in performing daily life activities compared to the control group $(\mathrm{p}=0.036$, Mann-Whitney U). If this comparison was made controlling for best corrected vision, better eye, the AMD subjects still perceived significantly more difficulties compared to the control group if the VA was $<0.6$ (decimal notation). For better preoperative VA, there was no difference between the groups. After surgery the AMD group perceived significantly more difficulties in performing daily life activities both in all subjects and in the older ( $\geqslant 80$ years) subgroup $(p<0.001$, Mann-Whitney U). For both all subjects and the older subgroup this difference was most evident when the postoperative VA in the operated eye was in the interval $0.4-0.7$ (decimal notation).

The perceived difficulties to perform different daily life activities for both groups of patients before and after surgery are outlined in Table 7. The AMD subjects had more perceived difficulties in practically all daily life activities before surgery. However, the difference between the groups of subjects is only significant in three items for the 5\% level (reading paper text, seeing prices, and seeing text on TV). After surgery the AMD

Table 8 Comparison of disability scores for different activities before and after surgery for AMD subjects

\begin{tabular}{lll}
\hline & \multicolumn{2}{l}{$\begin{array}{l}\text { Comparison of scores } \\
\text { before and after surgery }\end{array}$} \\
\cline { 2 - 3 } Activity & Z value & $\mathrm{p} \mathrm{Value*}$ \\
\hline Reading paper text & -5.369 & $<0.001$ \\
Recognising faces & -3.920 & $<0.001$ \\
Seeing prices & -5.130 & $<0.001$ \\
Seeing to walk on uneven ground & -2.308 & 0.021 \\
Seeing needlework & -3.435 & 0.001 \\
Seeing text on TV & -4.265 & 0.001 \\
Seeing to perform a preferred hobby & -1.976 & 0.048 \\
\hline \multirow{2}{*}{ *Wilcoxon signed ranks test. } & & \\
\hline
\end{tabular}

group had significantly more perceived difficulties in all items $(\mathrm{p}<0.001$, Mann-Whitney U) (Table 7).

As can be seen in Table 7 the activities causing the highest degree of perceived difficulties were those where near vision is used (reading paper text, seeing prices, and doing needlework).

If the same tests were performed on the subgroup of patients aged 80 or older, the result was almost identical. The AMD group compared to the controls had significantly more perceived difficulties before surgery in two items: seeing prices and seeing text on TV. After surgery there were significantly more difficulties for the AMD group compared to the controls in all items. For all subjects with AMD there was a significant improvement in perceived difficulties in performing daily life activities after surgery (Table 8). As can be seen in Table 8 , "reading paper text," "seeing prices," and "seeing text on TV" were the activities that showed greatest improvement after surgery.

Tests of the relation between preoperative variables and outcome for AMD subjects are shown in Table 9. In these analyses, a number of preoperatively known variables were tested in a logistic regression model against three different dichotomised outcomes as dependent variables. A good preoperative VA in the fellow eye was related to an improvement in perceived difficulties in daily life. Second eye surgery and dissatisfaction with vision preoperatively were related to dissatisfaction with vision also after surgery (Table 9). First eye surgery of subjects with AMD who were dissatisfied with their vision before surgery resulted in dissatisfaction for $53.3 \%$ also after surgery. Detailed analysis of AMD subjects undergoing second eye surgery in our study showed that $77.8 \%$ of those who were dissatisfied with their vision preoperatively also ended up dissatisfied after surgery. Poor VA in the fellow eye (=previous cataract surgery eye) was also

Table 9 Relation ( $p$ value, logistic regression) between known preoperative variables and the dichotomised outcome for AMD subjects

\begin{tabular}{llll}
\hline & \multicolumn{3}{l}{ Dichotomised self assessed functional outcome } \\
\cline { 2 - 4 } Variables & $\begin{array}{l}\text { Improved or } \\
\text { status quo v } \\
\text { worse p value* }\end{array}$ & $\begin{array}{l}\text { Improved or } \\
\text { status quo } v \\
\text { worse p value* }\end{array}$ & $\begin{array}{l}\text { Satisfied } v \\
\text { dissatisfied } \\
\text { p value* }\end{array}$ \\
\hline Age & 0.829 & 0.883 & 0.076 \\
Sex & 0.795 & 0.661 & 0.203 \\
Second eye surgery & 0.471 & 0.722 & 0.007 \\
VA, fellow eye & 0.033 & 0.011 & 0.495 \\
Activity score & 0.318 & 0.354 & 0.877 \\
Disability score & 0.318 & 0.167 & 0.119 \\
Global score & 0.970 & 0.729 & 0.020 \\
\hline *Logistic regression. & \multicolumn{3}{l}{} \\
\hline
\end{tabular}




\begin{tabular}{|c|c|c|c|c|c|c|}
\hline & \multicolumn{6}{|l|}{ After surgery } \\
\hline & \multicolumn{3}{|l|}{ Control } & \multicolumn{3}{|l|}{ AMD } \\
\hline & No & Yes & Total & No & Yes & Total \\
\hline \multicolumn{7}{|c|}{ Before surgery } \\
\hline No & 252 & 10 & $262(82.4 \%)$ & 54 & 4 & 58 175.3\% \\
\hline Yes & 23 & 33 & $56(17.6 \%)$ & 4 & 15 & $19124.7 \%$ \\
\hline Total & $275(86.5 \%)$ & $43(13.5 \%)$ & 318 & 58 (75.3\%) & $19(24.7 \%)$ & 77 \\
\hline
\end{tabular}

related to a worse outcome (improved versus status quo or not improved: $p=0.018$, Mann-Whitney $U$; satisfied versus dissatisfied: $p=0.003$, Mann-Whitney $U$ ).

Thirty four subjects had AMD and a preoperative VA in the eye to be operated on of 0.1 or less. For those who after surgery still had a VA of 0.1 at the most $(n=11)$ only $27 \%(n=3)$ improved their perceived difficulties in performing daily life activities. On the other hand, those who improved their VA to 0.2 or better after surgery improved in perceived difficulties in $91 \%$ of cases (21 out of 23 ).

Cataract surgery also has an impact on independence. The number of subjects with home help before and after surgery is shown in Table 10. For subjects with no ocular comorbidity there was a significant decrease in use of home help after surgery ( $p=0.024$, Wilcoxon signed rank test), but for AMD subjects the use of home help was the same.

\section{DISCUSSION}

The study design was observational and prospective on a multicentre basis. Consecutive cases operated during 1 month were included from six different surgical departments. A weakness in this study was the loss (approximately 25\%) during follow up mainly because of incomplete postoperative questionnaires. Earlier studies have shown that subjects not answering the postoperative questionnaire are older and have a higher frequency of other diseases and handicaps. ${ }^{18}$ Obviously, a high number of AMD patients did not answer the postoperative questionnaire in this study (Table 1). Therefore, it is reasonable to believe that the outcome for AMD subjects might have been somewhat worse if all AMD subjects had been included.

One important feature of Catquest is that the questions refer to perceived difficulties in performing a daily life activity at the time of questioning and not to any perceived improvement after surgery. Nor are there any questions about satisfaction with care. It is generally known that many patients with AMD and cataract are grateful for having the chance of surgery and may even say that their vision improved after surgery though this can not be demonstrated by testing VA.

The AMD subjects in this study improved significantly in their visual function and satisfaction with vision after surgery. However, the improvement was not as good as for the control group. As found in other studies ${ }^{13}{ }^{14}$ the mean age of the AMD group was significantly higher. After adjustments of the difference in age there was a significantly better outcome for the control group.

The most important variable related to the outcome was postoperative VA in the operated eye. The level of the postoperative VA in the operated eye seems to be the most significant factor for a good functional outcome and satisfaction with vision after surgery, not the magnitude of improvement accomplished by surgery. Subjects with no ocular comorbidity have generally better VA in the operated eye after surgery than AMD subjects and this is the reason for a better functional outcome and satisfaction with vision among the former group. If the difference in postoperative VA in the operated eye was levelled out by constructing groups with equal postoperative VA, no difference in functional outcome between AMD subjects and control subjects was found (Table 6).

Looking at the difficulties in performing various daily life activities, AMD subjects clearly have more difficulties before surgery than control subjects. With regard to three items this difference is significant $(0.05 \%$ level $)$; seeing prices, reading paper text, and seeing text on TV. After surgery AMD subjects have significantly more difficulties than control subjects in all items. As a group, however, the AMD subjects significantly improved their perceived difficulties in performing daily life activities after surgery. Greatest improvement was found in activities which use near vision. This was also reported by Armbrecht et al. ${ }^{14}$

Predictors of poor outcome in our study were a low VA in the fellow eye and/or dissatisfaction with vision in subjects scheduled for second eye surgery. Obviously these subjects need to be carefully informed before possible surgery. An interesting group of subjects was the one with a low VA ( $\leqslant$ $0.1)$ in the eye to be operated on. If VA improved after surgery to 0.2 or more the self assessed functional outcome was extremely good. If the VA was unchanged after surgery very few improved their self assessed visual function. Information about VA before the development of cataract and the possibility to improve VA by surgery seem essential for this group of subjects.

There was no information about the severity of lens opacities in our study and the morphological changes in macula were not classified in stages. This may have disclosed other subgroups with a predictable outcome. However, studies have pointed out that there is no close relation between the degree of lens opacities and dissatisfaction with vision. ${ }^{19}$ Furthermore, the prevalence of AMD causing visual impairment is smaller than the prevalence of any visible changes in the macula. ${ }^{20}$ We believe, that the most important variables to support the decision of surgery in patients with AMD and cataract are not to be found in morphological changes of the eye, but rather in the perceived disabilities and visual function of the individual.

The disease specific instrument used in this study (Catquest) was originally designed for patients with cataract. Subjects in this study also suffered from AMD. No question to the subjects in our study focused on use of low vision aids or other activities related to a very poor vision. On the other hand, other instruments originally designed for cataract patients have also been successfully used in AMD patients..$^{22}$ Catquest too, appears useful to distinguish between outcomes of patients with a macular disease and other patients. However, questions about disabilities and activities not included in the questionnaire-for example, use of magnifying glass, or other magnifying devices, might have revealed benefits of cataract surgery not found in the study.

A question of concern, which lies outside the purpose of the present study, is whether a cataract extraction may worsen a 
macular degeneration. Findings supporting this hypothesis have been published. ${ }^{23}$

\section{Authors' affiliations}

M Lundström, Department of Ophthalmology, Blekinge Hospital, SE-371 85 Karlskrona, Sweden

K G Brege, Department of Ophthalmology, Lake Maelar Hospital,

SE-631 88 Eskilstuna, Sweden

I Florén, Department of Ophthalmology, Lund University Hospital, SE-221 85 Lund, Sweden.

B Lundh, Department of Ophthalmology, Linköping University Hospital, SE-581 85 Linköping, Sweden

U Stenevi, Department of Ophthalmology, Sahlgren's University Hospital, SE-431 80 Mölndal, Sweden.

W Thorburn, Umeå University Hospital, SE-901 85 Umeå, Sweden

\section{REFERENCES}

1 Lundstrom M, Stenevi U, Thorburn W. Age-related utilisation of cataract surgery in Sweden during 1992-1999. A retrospective study of cataract surgery rate in one-year age groups based on the Swedish National Cataract Register. Acta Ophthalmol Scand 2001;79:342-9.

2 Norregaard JC, Bernth-Petersen P, Folmer-Andersen T. Changing threshold for cataract surgery in Denmark between 1980 and 1992 Results from the Danish Cataract Surgery Outcomes Study. II. Acta Ophthalmol Scand 1996:74/6:604-8.

3 Taylor HR. Cataract: how much surgery do we have to do? $\mathrm{Br} J$ Ophthalmol 2000;84:1-2

4 Lundström M, Stenevi U, Thorburn W. Quality of life after first- and second-eye cataract surgery. Five-year data collected by the Swedish National Cataract Register. J Cataract Refract Surg 2001;27: 1553-9.

5 Laidlaw DAH, Harrad RA, Hopper CD, et al. Randomised trial of effectiveness of second eye cataract surgery. Lancet 1998;352:925-9.

6 Lundström M, Barry P, Leite E, et al. The 1998 European Cataract Outcome Study. Report from the European Cataract Outcome Study. J Cataract Refract Surg 2001;27: 1 176-84.

7 Cataract Management Guideline Panel. Cataract in adults: management of functional impairment. Rockville, MD: US Department of Health and Human Services, Public Health Service, Agency for Health Care Policy and Research, 1993 (AHCPR pub no93-0542; Clinical practice guideline; no 4).
8 Schein OD, Steinberg EP, Cassard S, et al. Predictors of outcome in patients who underwent cataract surgery. Ophthalmology $1995 ; 102: 817-23$

9 Lundström M, Stenevi U, Thorburn W. Outcome of cataract surgery considering the preoperative situation: a study of possible predictors of the functional outcome. Br J Ophthalmol 1999;83:1272-6.

10 Lundström $M$, Brege KG, Florén I, et al. Impaired visual function after cataract surgery assessed using the Catquest questionnaire. I Cataract Refract Surg 2000;26:101-8.

11 Tielsch JM, Steinberg EP, Cassard SD, et al. Preoperative functional expectations and postoperative outcomes among patients undergoing first eye cataract surgery. Arch Ophthalmol 1995;113:1312-18.

12 Westcott MC, Tuft SJ, Minassian DC. Effect of age on visual outcome following cataract extraction. Br J Ophthalmol 2000;84:1380-2

13 Shuttleworth GN, Luhishi EA, Harrad RA. Do patients with age related maculopathy and cataract benefit from cataract surgery? Br J Ophthalmol
moth 1998;82:611-16.

14 Armbrecht AM, Findlay C, Kaushal S, et al. Is cataract surgery justified in patients with age related macular degeneration? A visual function and quality of life assessment. Br J Ophthalmol 2000;84:1343-8.

15 Lundström M, Stenevi U, Thorburn W. The Swedish National Cataract Register B. A nine-year review. Acta Ophthalmol Scand 2002;80:248-57.

16 Holladay JT, Prager C. Mean visual acuity (Letter). Am J Ophthalmol 1991;111:372-4.

17 Lundström M, Roos $\mathrm{P}$, Jensen $\mathrm{S}$, et al. Catquest questionnaire for use in cataract surgery care: Description, validity and reliability. J Cataract Refract Surg 1997:23:1226-36.

18 Lundström M, Stenevi U, Thorburn W, et al. Catquest questionnaire for use in cataract surgery care: Assessment of surgical outcomes. J Cataract Refract Surg 1998;24:968-74.

19 McCarty CA, Keeffe JE, Taylor HR. The need for cataract surgery: projections based on lens opacity, visual acuity, and personal concern. Br J Ophthalmol 1999:83:62-5.

20 Laatikainen L, Hirvelä $\mathrm{H}$. Prevalence and visual consequences of macular changes in a population aged 70 years and older. Acta Ophthalmol Scand 1995;73:105-10

21 Mangione CM, Gutierrez PR, Lowe G, et al. Influence of age-related maculopathy on visual functioning and health-related quality of life. Am J Ophthalmol 1999;128:45-53.

22 Linder M, Chang TS, Scott IU, et al. Validity of the Visual Function Index (VF-14) in patients with retinal disease. Arch Ophthalmol 1999;117:1611-16

23 Pollack A, Marcovitch A, Bukelman A, et al. Development of exudative age-related macular degeneration after cataract surgery. Eye $1997 ; 11: 523-30$.

\section{Video Reports (www.bjophthalmol.com)}

- Capsule staining and mature cataracts: a comparison of indocyanine green and trypan blue dyes. D F Chang

- Pearls for implanting the Staar toric IOL. D F Chang

- An intraocular steroid delivery system for cataract surgery. D F Chang

- Evaluation of leucocyte dynamics in mouse retinal circulation with scanning laser ophthalmoscopy. $H X_{U}$, A Manivannan, G Daniels, J Liversidge, P F Sharp, J V Forrester, IJ Crane

- Dipetalonema reconditum in the human eye. T Huynh, J Thean, R Maini

- Surgical revision of leaking filtering blebs with an autologous conjunctival graft. K Taherian, A Azuara-Blanco

- Thixotropy: a novel explanation for the cause of lagophthalmos after peripheral facial nerve palsy. M Aramideh, J H T M Koelman, P P Devriese, F VanderWerf, J D Speelman 